\title{
Retraction
}

\section{Culture as a variable in neuroscience and clinical neuropsychology}

\section{A comprehensive review}

José Roberto Wajman, Paulo Henrique Ferreira Bertolucci, Letícia Lessa Mansur, Serge Gauthier

The editorial board of the journal Dementia $\mathcal{E}$ Neuropsychologia communicates the formal retraction of the article:

Culture as a variable in neuroscience and clinical neuropsychology: a comprehensive review. José Roberto Wajman, Paulo Henrique Ferreira Bertolucci, Letícia Lessa Mansur, Serge Gauthier. Dement Neuropsychol 2015; 9(3):203-218.

This article is being retracted due to the identification of passages without due citation and others reproduced verbatim without quotation marks. The authors recognize these issues but the first author attributes them to erroneous submission of the wrong electronic version. 